\title{
Relação entre atividade física e densidade mineral óssea/osteoporose: uma revisão da literatura nacional
}

\author{
Heberth Rocha Barros \\ Raphael Mendes Ritti-Dias \\ Escola Superior de Educação Física da Universidade de Pernambuco, Recife, PE, Brasil
}

\begin{abstract}
Resumo: O objetivo deste estudo foi revisar a literatura nacional sobre atividade física e densidade mineral óssea (DMO)/osteoporose. Foi realizada pesquisa bibliográfica nas bases de dados Lilacs e Sibradid. Foram utilizados os termos "osteoporose" e "densidade óssea" que foram combinados com os termos "atividade física" e "exercício físico". Foram incluídos os estudos originais que foram publicados em língua portuguesa, relacionavam a DMO ou osteoporose com atividade física ou exercício físico em humanos e estavam disponíveis online. Sete estudos atenderam a esses critérios e foram incluídos na revisão. Dos três estudos que analisaram a associação entre a prática de atividade física na infância e a DMO/osteoporose, apenas um verificou associação significante. Dos seis estudos que analisaram a relação entre a atividade física atual e a $\mathrm{DMO}$, quatro estudos verificaram relação significante. Os resultados dessa revisão evidenciaram a existência de poucos estudos que investigaram a relação entre atividade física e DMO/osteoporose na literatura nacional. Os resultados destes estudos, embora apresentem algumas controvérsias, sugerem que a atividade física pode aumentar a DMO e diminuir a prevalência de osteoporose.
\end{abstract}

Palavras-chave: Exercício. Atividade motora. Densidade óssea.

\section{Relationship between physical activity and bone density/osteoporosis: a review of the brazilian literature}

\begin{abstract}
The objective of this study was to review the Brazilian literature on physical activity/exercise and bone mineral density (BMD)/ osteoporosis. A bibliographic search was performed at Lilacs and Sibradid databases. The terms "osteoporosis" and "bone density" were combined with "physical activity" and "exercise". Original studies that related BMD or osteoporosis with physical activity or exercise in humans, in Portuguese and available online were included. Seven studies met these criteria and were included in the review. Out of three studies that analyzed the association between physical activity practice in childhood and the BMD/osteoporosis, only one found significant association. Four out of six studies that analyzed the relationship between actual physical activity/exercise with BMD showed significant relation. The results of this systematic review demonstrated that few studies investigate the relation between physical activity/ exercise and BMD/ osteoporosis in the Brazilian literature. Although the results present some controversies, they suggest a positive impact on physical activity/exercise to increase BMD and decrease the prevalence of osteoporosis.
\end{abstract}

Key Words: Exercise. Motor activity. Bone density.

\section{Introdução}

A osteoporose é uma das doenças crônicodegenerativas mais comuns no envelhecimento (KAUFFMAN, 2001). Esse distúrbio osteometabólico é caracterizado pela diminuição da densidade mineral óssea (DMO), com deterioração da microarquitetura óssea, levando a um aumento da fragilidade esquelética e do risco de fraturas (PINTO NETO et al., 2002; AVEIRO et al., 2004; NAVEGA et al., 2006; NAVEGA;OISHI, 2007; AUAD et al., 2008), que, por sua vez, tem sido associado ao aumento da morbidade e mortalidade entre os idosos (PINTO NETO et al., 2002). Além das conseqüências físicas, esta doença tem sido associada a problemas psicológicos que fazem da osteoporose um dos maiores problemas de saúde pública da atualidade (JACKA et al., 2005).

Ao longo das últimas décadas, vários tratamentos têm sido propostos objetivando ao aumento ou atenuação da perda da DMO (LIU;LEBRUN, 2006; ZEHNACKER;BEMISDOUGHERTY, 2007; KASTURI et al., 2009). Entre eles estão a terapia de reposição hormonal, o uso de compostos bifosfonatos e os programas de exercício físico (PINTO NETO et al., 2002). Mais especificamente com relação à prática de atividade física, a força mecânica, que ocorre durante o exercício físico tem sido considerada 
como importante estímulo para a osteogênese (TENÓRIO et al., 2005; KEMPER et al., 2009).

A prática de atividade física ao longo de diferentes fases da vida tem sido sugerida como fator de proteção de osteoporose (HASSELSTROM et al., 2007; MIYABARA et al., 2007; NILSSON et al., 2008). De fato, alguns estudos epidemiológicos transversais(HASSELSTROM et al., 2007; MIYABARA et al., 2007; NILSSON et al., 2008) analisando a influência da prática de atividade física na infância ou na idade adulta têm sugerido que os indivíduos mais ativos fisicamente são aqueles que apresentam maior DMO. Esses achados também vêm sendo corroborados por estudos longitudinais que verificaram efeitos positivos do treinamento físico da DMO de humanos e modelos experimentais(JUDGE et al., 2005; KATO et al., 2006).

Por outro lado, alguns estudos não têm evidenciado aumento da DMO com os programas de exercício físico (KERSCHAN-SCHINDL et al., 2000; LANZILLOTTI et al., 2003; CHAN et al., 2004; MOSER et al., 2004). Embora os protocolos de exercício físico utilizados nesses estudos venham sendo apontados como a principal causa do não aumento da $\mathrm{DMO}$, outros fatores também parecem influenciar os efeitos do treinamento físico na DMO (HOUTKOOPER et al., 1995; SHIGUEMOTO et al., 2007), o que poderia também contribuir para essa controvérsia.

De fato, tem sido demonstrado que os aumentos da DMO com o treinamento físico podem ser influenciados pelo estilo de vida da amostra estudada (EISMAN et al., 1991; HOUTKOOPER et al., 1995; PRINCE et al., 1995; WELCH;WEAVER, 2005; SHIGUEMOTO et al., 2007). Nesse sentido, estudos em populações com características sócio-culturais diferentes, e, consequentemente, estilos de vida e hábitos alimentares diferentes, podem ter contribuido para a controvérsia entre os estudos. Dessa forma, a análise dos efeitos do treinamento físico na DMO em cada subgrupo populacional poderia minimizar esse viés. Diante disso, o objetivo deste estudo foi revisar a literatura nacional sobre a relação atividade física, DMO e osteoporose.

\section{Métodos}

Foi realizada revisão a partir da pesquisa bibliográfica de artigos originais sobre a prática de atividades físicas/exercício físico e DMO/osteoporose, incluindo estudos publicados de 1985 até maio de 2009. A pesquisa bibliográfica realizada nas bases de dados Lilacs e Sibradid incluiu somente artigos desenvolvidos no Brasil e no idioma português.

Para realização da pesquisa, foram utilizados os termos "osteoporose" e "densidade óssea" que foram combinados com os termos "atividade física" e "exercício físico". Foram incluídos os artigos publicados em língua portuguesa, que relacionavam a DMO ou osteoporose com atividade física ou exercício físico em seres humanos e que estavam disponíveis online.

Para análise do atendimento aos critérios de inclusão, foi feita inicialmente a leitura do título e dos resumos. Na impossibilidade de que esta decisão fosse tomada pela leitura do título e resumo, recorreu-se a leitura do artigo na íntegra. Não foram incluídos artigos de revisão, estudos de validação, monografias, dissertações e teses.

Todos os artigos obtidos foram analisados por dois pesquisadores que preencheram um quadro síntese incluindo os seguintes aspectos: (a) número de sujeitos na amostra; (b) características da amostra; (c) variáveis analisadas; (d) delineamento experimental; (e) instrumento de medida da atividade física; (f) instrumento para a medida da DMO/Osteoporose; (g) análise estatística empregada; (h) principais resultados.

\section{Resultados}

Com os termos utilizados na presente estudo foram encontrados ao todo 83 estudos. Destes, 29 eram revisão, 25 não analisavam a prática de atividade física ou exercício físico e cinco eram monografias, dissertações ou teses; e não adentraram nesta revisão.

Dos 24 estudos restantes, oito não estavam disponíveis em formato online, três foram realizados em modelos experimentais, cinco analisaram os efeitos da atividade física/exercício físico na qualidade de vida ou aptidão física e não analisaram DMO ou osteoporose e um estudo não fornecia informações sobre os métodos utilizados para o diagnóstico de atividade física e da densidade mineral óssea, portanto, não foram incluídos nesta revisão.

No final, sete estudos foram incluídos nesta revisão. Na tabela 1 é apresentado o resumo dos estudos incluídos na revisão. 
Para apresentação dos resultados, os estudos foram agrupados de acordo com o tema em: estudos que analisaram associação entre a prática de atividade física na infância e a DMO ou osteoporose e estudos que analisaram a associação entre a prática de atividade física/exercício físico atual e a DMO.

Tabela 1. Resumo dos estudos incluídos na revisão.

\begin{tabular}{lccl}
\hline \multicolumn{1}{c}{ Autor } & \multicolumn{1}{c}{ Amostra } & Delineamento & Variáveis analisadas \\
\hline Cesar et al & 17 Mulheres 24-40 anos & Transversal & Densidade mineral óssea \\
Frazão et al & 413 Mulheres 30-85 anos & Transversal & Densidade mineral óssea \\
Lanzillotti et al. & 76 mulheres 46-85 anos & Transversal & Presença de osteoporose \\
Mesquita et al & 60 Mulheres 13-17 anos & Transversal & Densidade mineral óssea \\
Moser et al & 200 Mulheres 36-76 anos & Transversal & Densidade mineral óssea \\
$\underline{\text { Pires et al }}$ & 46 Meninos 7-13 anos & Transversal & Densidade mineral óssea \\
Siqueira et al & 1016 Homens e mulheres & Transversal & Presença de osteoporose \\
& com mais de 50 anos & &
\end{tabular}

Três estudos analisaram a associação entre a prática de atividade física na infância e adolescência e a DMO ou osteoporose (tabela 2).

Tabela 2. Associação entre a prática de atividade física/exercício na infância e a densidade mineral óssea ou a presença de osteoporose.

\begin{tabular}{|c|c|c|c|c|}
\hline Estudo & Medida AF & $\begin{array}{c}\text { Medida } \\
\text { DMO/Osteoporose }\end{array}$ & Análise estatística & Resultados \\
\hline Lanzillotti et al & $\begin{array}{c}\text { Questões semi } \\
\text { estruturadas sobre } \\
\text { prática de AF }\end{array}$ & DEXA & Não relatado & $\begin{array}{l}\text { AF na juventude não teve } \\
\text { associação com a DMO atual } \\
\text { OR=1,00; IC= } 0,40-2,54\end{array}$ \\
\hline Moser et al & $\begin{array}{l}\text { Questões semi } \\
\text { estruturadas sobre } \\
\text { prática de AF }\end{array}$ & DEXA & Teste qui-quadrado & $\begin{array}{l}\text { AF na infância não teve } \\
\text { associação com a DMO } \\
X^{2}=2,8\end{array}$ \\
\hline$\underline{\text { Siqueira et al }}$ & $\begin{array}{l}\text { Questões semi } \\
\text { estruturadas sobre } \\
\text { prática de AF }\end{array}$ & Diagnóstico prévio & $\begin{array}{c}\text { Análise multivariada } \\
\text { através da regressão } \\
\text { de Poisson }\end{array}$ & $\begin{array}{c}\text { AF adolescência associou-se } \\
\text { menor prevalência de } \\
\text { osteoporose } \\
\mathrm{OR}=0,56 ; \mathrm{IC}=0,36-0,86\end{array}$ \\
\hline
\end{tabular}

AF - atividade física; DEXA- Absortometria Radiológica de Dupla-Energia; DMO - Densidade mineral óssea; OR Razão de chance; IC - intervalo de confiança.

Dos três estudos, apenas um (SIQUEIRA et al., 2009) verificou associação significante entre o nível de atividade física na infância e adolescência e a presença de osteoporose.

Seis estudos investigaram a associação entre a prática atual de atividade física/exercício físico e a DMO (tabela 3).

Dos seis estudos que analisaram a associação entre a prática de atividade física/exercício físico atual e a DMO, quatro verificaram associação significante(CESAR et al., 2001; PIRES et al., 2005; MESQUITA et al., 2008; FRAZÃO;NAVEIRA, 2007), ao passo que dois estudos não evidenciaram associação entre a prática de atividade física e a DMO. Nos estudos que observaram associação significante a amostra foi composta de mulheres adolescentes, mulheres jovens, mulheres idosas e crianças e adolescentes do sexo masculino. 
Tabela 3. Associação entre a prática de atividade física/exercício físico atual e a densidade mineral óssea

\begin{tabular}{|c|c|c|c|c|}
\hline Estudo & Medida AF & $\begin{array}{l}\text { Medida } \\
\text { DMO }\end{array}$ & Análise Estatística & Resultados \\
\hline$\underline{\text { Cesar et al }}$ & $\begin{array}{l}\text { Prática regular de } \\
\text { corrida }\end{array}$ & DEXA & $\begin{array}{l}\text { Teste t para } \\
\text { amostras } \\
\text { independentes }\end{array}$ & $\begin{array}{c}\text { Corredoras apresentam maior DMO } \\
\text { DMO coluna }\left(\mathrm{g} / \mathrm{cm}^{2}\right) \\
1,3 \pm 0,1^{*} \text { vs. } 1,2 \pm 0,1 \\
\\
\text { DMO fêmur }\left(\mathrm{g} / \mathrm{cm}^{2}\right) \\
1,1 \pm 0,2^{*} \text { vs, } 0,9 \pm 0,1\end{array}$ \\
\hline Frazão et al & $\begin{array}{l}\text { Análise de } \\
\text { prontuário }\end{array}$ & DEXA & $\begin{array}{l}\text { Regressão logística } \\
\text { múltipla não } \\
\text { condicional }\end{array}$ & $\begin{array}{c}\text { Maior nível } \mathrm{AF} \text { foi fator protetor para } \\
\text { baixa } \mathrm{DMO} \\
\text { OR }=0,47^{*} ; \mathrm{IC}=0,23 ; 0,97\end{array}$ \\
\hline Lanzillotti et al. & $\begin{array}{l}\text { Questões semi } \\
\text { estruturadas sobre } \\
\text { prática de AF }\end{array}$ & DEXA & Não relatado & $\begin{array}{l}\text { AF atual não teve associação com a } \\
\text { DMO } \\
\text { OR=1,15; IC=0,46 }-2,8\end{array}$ \\
\hline Mesquita et al & $\begin{array}{l}\text { Prática regular de } \\
\text { voleibol }\end{array}$ & DEXA & $\begin{array}{l}\text { Teste t para } \\
\text { amostras } \\
\text { independentes }\end{array}$ & $\begin{array}{c}\text { Praticantes de voleibol apresentam } \\
\text { maior DMO } \\
\text { DMO corpo inteiro }\left(\mathrm{g} / \mathrm{cm}^{2}\right) 1,2 \pm 0,1 \\
\text { vs. } 1,1 \pm 0,1^{*} \\
\text { DMO fêmur }\left(\mathrm{g} / \mathrm{cm}^{2}\right) \\
1,2 \pm 0,1 \text { vs. } 1,0 \pm 0,1^{*}\end{array}$ \\
\hline & & & & $\begin{array}{c}\text { DMO triâng. Wards }\left(\mathrm{g} / \mathrm{cm}^{2}\right) \\
1,1 \pm 0,1 \text { vs. } 0,9 \pm 0,2^{*}\end{array}$ \\
\hline$\underline{\text { Moser et al }}$ & $\begin{array}{l}\text { Questões semi } \\
\text { estruturadas sobre } \\
\text { prática de AF }\end{array}$ & DEXA & Teste qui-quadrado & $\begin{array}{c}\text { A prática de AF não teve influência } \\
\text { na DMO } \\
x^{2}=0,22\end{array}$ \\
\hline$\underline{\text { Pires et al }}$ & $\begin{array}{l}\text { Questões sobre AF } \\
\text { escolares, de lazer } \\
\text { e extra-escolares }\end{array}$ & DEXA & $\begin{array}{l}\text { Teste t para } \\
\text { amostras } \\
\text { independentes }\end{array}$ & $\begin{array}{c}\text { Meninos que sofreram fraturas } \\
\text { apresentaram menor prevalência de } \\
\text { AF extra-escolar } \\
26,1 \% \text { vs } 65,2 \%^{*}\end{array}$ \\
\hline
\end{tabular}

\section{Discussão}

Os resultados dos estudos apresentados nesta revisão indicam que a literatura nacional apresenta resultados controversos sobre a relação entre a prática de atividade física e a DMO e osteoporose. Essa controvérsia foi observada tanto na relação da atividade física/exercício físico praticado na infância e na adolescência, como na atividade física atual e a DMO ou osteoporose.

Dos três estudos que analisaram a relação entre a prática de atividade física na infância e adolescência sobre a DMO e a prevalência de osteoporose, apenas um (SIQUEIRA et al., 2009) evidenciou que a atividade física na infância está associada à menor prevalência de osteoporose na idade adulta, ao passo que os outros estudos não evidenciaram associação significante (LANZILLOTTI et al., 2003; MOSER et al., 2004). Parte dessa controvérsia pode ter sido ocasionada pela análise estatística empregada. Enquanto o estudo de Siqueira et al. (2003) utilizou análise multivariada, os outros estudos ou utilizaram análise bivariada, ou não reportaram a análise empregada. Esse é um aspecto relevante, uma vez que a análise multivariada é o procedimento mais indicado para análise desse tipo de associação, uma vez que permite controlar possíveis fatores de confusão, como por exemplo, o peso corporal, idade, cor da pele, nível sócio econômico, entre outros. De fato, estudos disponíveis na literatura internacional que analisaram a associação entre a prática de atividade física na infância e a DMO e que analisaram os dados com estatística multivariada evidenciaram que o maior nível de atividade física na infância está associado à maior DMO na idade adulta (FLORINDO et al., 2002; FORD et al., 2004; BARNEKOW-BERGKVIST et al., 2006).

A maioria dos estudos nacionais que investigaram a associação entre a prática de atividade física/exercício físico atual e a DMO sugere que 0 maior nível de atividade física/exercício físico atual está associado à maior DMO em mulheres de diferentes níveis maturacionais. A associação entre a prática de atividade física e maior DMO foi observada em crianças, adolescentes, jovens e idosas que realizaram diferentes modalidades, o que está em linha com estudos internacionais sobre essa 
temática (CHENG et al., 1991; BURROWS ARGOTE et al., 1996; DALY et al., 2008). Por outro lado, dois estudos não evidenciaram associação entre a prática de atividade física atual e a DMO. Interessantemente, os dois estudos que não evidenciaram essa associação são os mesmos que também não evidenciaram associação entre a prática de atividade física na infância e a DMO.

É importante ressaltar que o delineamento transversal empregado nos estudos nacionais que analisaram a relação entre a prática de atividade física atual e a DMO, consiste em limitação extremamente importante, uma vez que esse delineamento não permite estabelecer uma relação de causa e efeito. Dessa forma, é possível que as crianças, adolescentes, mulheres de meia idade e idosas ativas já apresentassem maior DMO antes do início da prática de atividade física que estava sendo realizada atualmente, de forma que a maior DMO nesses indivíduos não tenha sido decorrente da prática atual de atividade física. Embora alguns dos estudos tenham buscado controlar fatores de confusão tais como a ingestão de cálcio, o peso corporal e a utilização de hormônios, outros fatores importantes, como, por exemplo, a prática de atividade física no passado, na maioria das vezes não foi controlada. Interessantemente, a pesquisa bibliográfica realizada só identificou estudos longitudinais em modelos experimentais. Nesse sentido, sugere-se a realização de estudos nacionais em humanos com delineamento longitudinal que permitam identificar os efeitos do exercício físico na DMO de diferentes populações.

Com base nos estudos apresentados nessa revisão, é possível perceber há existência de muitas lacunas de conhecimento sobre a relação entre atividade física/exercício físico e DMO. Por exemplo, há escasso número de estudos longitudinais sobre essa temática. Além disso, ainda existem poucas evidências sobre a comparação de diferentes modalidades de exercício sobre a DMO/osteoporose, bem como a relação dose-efeito entre a prática de atividade física e os níveis de $\mathrm{DMO}$ e a prevalência de osteoporose.

\section{Conclusões}

Os resultados dessa revisão evidenciaram a existência de poucos estudos que investigaram a relação entre atividade física/exercício físico e DMO/osteoporose na literatura nacional. Os resultados destes estudos, embora apresentem algumas controvérsias, sugerem, em sua maioria, impacto positivo da atividade física para aumentar a DMO e diminuir a prevalência de osteoporose.

\section{Referências}

AUAD, M. A.;SIMÕES, R. P.;ROUHANI, S.;CASTELLO, V. ;YOGI, L. S. Eficácia de um programa de exercícios físicos na qualidade de vida de mulheres com osteoporose. Revista

Arquivos de Ciências da Saúde, Santo André, v. 33, n.1, p.31-35. 2008. Disponível em: http://lildbi.bireme.br/lildbi/docsonline/lilacs/20090 300/181-LILACS-UPLOAD.pdf. Acesso em: 17 nov 2009

AVEIRO, M. C.;NAVEGA, M. T.;GRANITO, R. N. ;RENNO, A. C. M. Efeitos de um programa de atividade física no equilíbrio e na força muscular do quadríceps em mulheres osteoporóticas visando uma melhoria na qualidade de vida. Revista Brasileira de Ciência e Movimento, Brasilia, v. 12, n.3, p.33-38. 2004. Disponível em: http://www.ucb.br/mestradoef/RBCM/12/12\%20\%203/c 123 5.pdf. Acesso em 17 nov 2009

BARNEKOW-BERGKVIST, M.;HEDBERG, G.;PETTERSSON, U. ;LORENTZON, R. Relationships between physical activity and physical capacity in adolescent females and bone mass in adulthood. Scandinavian Journal of Medicine and Science in Sports, Copenhagen, v. 16, n.6, Dec, p.447-55. 2006. doi: 10.1111/j.1600-0838.2005.00500.x

BURROWS ARGOTE, R.;LEIVA BALICH, L.;LILLO GANTER, R.;PUMARINO CARTE, H.;MAYA CASTILLO, L. ;MUZZO BENAVIDES, S. Influence of physical activity upon bone mineralization of school age children of both sexes. Archivos Latinoamericanos de Nutrición, Caracas, v. 46, n.1, Mar, p.11-5. 1996.

CESAR, M. C.;PARDINI, D. P. ;BARROS, T. L. Efeitos do exercício de longa duraçäo no ciclo menstrual, densidade óssea e potência aeróbica de corredoras. Revista Brasileira de Ciência e Movimento, Brasilia, v. 9, n.2, p.7-13. 2001. Disponível em:

http://www.ucb.br/mestradoef/RBCM/9/9\%20\%202/completo/c 92 1.pdf. Acesso em 17 nov 2009

CHAN, K.;QIN, L. ;LAU, M. A randomized, prospective study of the effect of Tai Chi Chun exercise on bone mineral density in postmenopausal women. Archives of Physical Medicine and Rehabilitation, Chicago, v. 85, p.717-22. 2004. doi:10.1016/j.apmr.2003.08.091

CHENG, S.;SUOMINEN, H.;RANTANEN, T.;PARKATTI, T. ;HEIKKINEN, E. Bone mineral density and physical activity in 50-60-year-old women. Journal of Bone and Mineral Research, Amsterdam, v. 12, n.2, Feb, p.123-32. 1991.

DALY, R. M.;AHLBORG, H. G.;RINGSBERG, K.;GARDSELL, P.;SERNBO, I. ;KARLSSON, M. 
K. Association between changes in habitual physical activity and changes in bone density, muscle strength, and functional performance in elderly men and women. Journal of the American Geriatrics Society, New York v. 56, n.12, Dec, p.2252-60. 2008. doi: 10.1111/j.15325415.2008.02039.x

EISMAN, J. A.;SAMBROOK, P. N.;KELLY, P. J. ;POCOCK, N. A. Exercise and its interaction with genetic influences in the determination of bone mineral density. American Journal of Medicine, New York, v. 91, n.5B, Nov 25, p.5S-9S. 1991.

FLORINDO, A. A.;LATORRE MDO, R.;JAIME, P. C.;TANAKA, T.;PIPPA, M. G. ;ZERBINI, C. A. Past and present habitual physical activity and its relationship with bone mineral density in men aged 50 years and older in Brazil. Journals A:

Biological Sciences and Medical Sciences, Washington, v. 57, n.10, Oct, p.M654-7. 2002. Disponível em:

http://biomed.gerontologyjournals.org/cgi/content/f ull/57/10/M654 Acesso em 17 nov 2009

FORD, M. A.;BASS, M. A.;TURNER, L. W.;MAUROMOUSTAKOS, A. ;GRAVES, B. S. Past and recent physical activity and bone mineral density in college-aged women Journal of Strength and Conditioning Research, Champaign, v. 18, n.3, Aug, p.405-9. 2004. doi: $10.1519 / 13343.1$

FRAZÃO, P. ;NAVEIRA, M. Fatores associados à baixa densidade mineral óssea em mulheres brancas. Revista de Saúde Pública, São Paulo, v. 41, n.5, p.740-748. 2007. Disponível em: http://www.scielo.br/pdf/rsp/v41n5/5910.pdf. Acesso em 17 nov 2009

HASSELSTROM, H.;KARLSSON, K. M.;HANSEN, S. E.;GRONFELDT, V.;FROBERG, K. ;ANDERSEN, L. B. Peripheral bone mineral density and different intensities of physical activity in children 6-8 years old: the Copenhagen School Child Intervention study. Calcified Tissue International, New York, v. 80, n.1, Jan, p.31-8. 2007. doi: 10.1007/s00223-006-0137-9

HOUTKOOPER, L. B.;RITENBAUGH, C.;AICKIN, M.;LOHMAN, T. G.;GOING, S. B.;WEBER, J. L.;GREAVES, K. A.;BOYDEN, T. W.;PAMENTER, R. W. ;HALL, M. C. Nutrients, body composition and exercise are related to change in bone mineral density in premenopausal women. Journal of Nutrition, Philadelphia, v. 125, n.5, May, p.1229-37. 1995.

JACKA, F. N.;PASCO, J. A.;HENRY, M. J.;KOTOWICZ, M. A.;DODD, S.;NICHOLSON, G. C. ;BERK, M. Depression and bone mineral density in a community sample of perimenopausal women: Geelong Osteoporosis Study.

Menopause, New York, v. 12, n.1, Jan-Feb, p.8891. 2005. doi: 00042192-200512010-00015
JUDGE, J. O.;KLEPPINGER, A.;KENNY, A.;SMITH, J. A.;BISKUP, B. ;MARCELLA, G. Home-based resistance training improves femoral bone mineral density in women on hormone therapy. Osteoporosis International, London, v. 16, n.9, Sep, p.1096-108. 2005. doi: $10.1007 / \mathrm{s} 00198-004-1816-x$

KASTURI, K. S.;CHENNAREDDYGARI, S. ;MUMMADI, R. R. Effect of bisphosphonates on bone mineral density in liver transplant patients: a meta-analysis and systematic review of randomized controlled trials. Transplant International, Oxford, v., Sep 30. 2009. 10.1111/j.1432-2277.2009.00976.x

KATO, T.;TERASHIMA, T.;YAMASHITA, T.;HATANAKA, Y.;HONDA, A. ;UMEMURA, Y. Effect of low-repetition jump training on bone mineral density in young women. Journal of Applied Physiology, Washington, v. 100, n.3, Mar, p.839-43. 2006.

doi:10.1152/japplphysiol.00666.2005

\section{KAUFFMAN, T. L. Manual de Reabilitação}

Geriátrica. Rio de Janeiro: Guanabara Koogan. 2001

KEMPER, C.;OLIVEIRA, R. J.;BOTTARO, M.;MORENO, R.;BEZERRA, L. M. A.;GUIDO, M. ;FRANÇA, N. M. Efeitos da natação e do treinamento resistido na densidade mineral óssea de mulheres idosas. Revista Brasileira de

Medicina do Esporte, Niterói, v. 15, n.1, p.10-13. 2009.

KERSCHAN-SCHINDL, K.;UHER,

E.;KAINBERGER, F.;KAIDER, A.;GHANEM, A.-H. ;PREISINGER, E. Long-term home exercise program: effect in women at high risk of fracture.

Archives of Physical Medicine and

Rehabilitation, Chicago, v. 81, p.319-23. 2000. doi:10.1016/S0003-9993(00)90078-9

LANZILLOTTI, H. S.;LANZILLOTTI, R. S.;TROTTE, A. P. R.;DIAS, A. S.;BORNAND, B. ;COSTA, E. A. M. M. Osteoporose em mulheres na pós-menopausa, cálcio dietético e outros fatores de risco. Revista de Nutrição, Campinas, v. 16, n.2, p.181-193. 2003. doi: 10.1590/S141552732003000200005

LIU, S. L. ;LEBRUN, C. M. Effect of oral contraceptives and hormone replacement therapy on bone mineral density in premenopausal and perimenopausal women: a systematic review.

British Journal of Sports Medicine,

Loughborough, v. 40, n.1, Jan, p.11-24. 2006. doi: 10.1136/bjsm.2005.020065

MESQUITA, W. G.;FONSECA, R. M. C. ;FRANÇA, N. M. Influência do voleibol na densidade mineral ossea de adolescentes do sexo feminino. Revista Brasileira de Medicina do Esporte, Niterói, v. 14, n.6, p.500-503. 2008. doi: 10.1590/S1517-86922008000600005. 
MIYABARA, Y.;ONOE, Y.;HARADA, A.;KURODA, T.;SASAKI, S. ;OHTA, H. Effect of physical activity and nutrition on bone mineral density in young Japanese women. Journal of Bone and Mineral Research, Tokyo, v. 25, n.6, p.414-8. 2007. doi: 10.1007/s00774-007-0780-x

MOSER, D. C.;MELO, S. I. L. ;SANTOS, S. G. Influência da atividade física sobre a massa óssea de mulheres. Revista Brasileira de Cineantropometria e Desempenho Humano, Florianópolis, v. 6, n.1, p.46-53. 2004. Disponível em:

http://www.rbcdh.ufsc.br/DetalhesArtigo.do?artigo $=111$. Acesso em 17 nov 2009

NAVEGA, M. T.;AVEIRO, M. C. ;OISHI, J. A influência de um programa de atividade física na qualidade de vida de mulheres com osteoporose. Revista Fisioterapia em Movimento, Curitiba, v. 19, n.4, p.25-32. 2006.

NAVEGA, M. T. ;OISHI, J. Comparação da qualidade de vida relacionada à saúde entre mulheres na pós-menopausa praticantes de atividade física com e sem osteoporose. Revista Brasileira de Reumatologia, São Paulo, v. 47, n.4, p.258-264. 2007.

NILSSON, M.;OHLSSON, C.;ERIKSSON, A. L.;FRANDIN, K.;KARLSSON, M.;LJUNGGREN, O.;MELLSTROM, D. ;LORENTZON, M. Competitive physical activity early in life is associated with bone mineral density in elderly Swedish men. Osteoporosis International, London, v. 19, n.11, Nov, p.1557-66. 2008. doi: $10.1007 / \mathrm{s} 00198-008-0600-8$

PINTO NETO, M. A.;SOARES, A.;URBANETZ, A A.;SOUZA, A. C. A.;FERRARI, A. E. M.;AMARAL, B.;MOREIRA, C.;FERNANDES, C. E.;ZERBINI, C. A. F.;BARACAT, E. ;FREITAS, E. C. Consenso Brasileiro de Osteoporose. Revista Brasileira de Reumatologia, São Paulo, v. 42, n.6, p.343-354. 2002.

PIRES, L. A. S.;SOUZA, A. C. A.;LAITANO, O. ;MEYER, F. Densidade mineral óssea, ingestão de leite e atividade física de meninos que sofreram fraturas no antebraco. Journal of Pediatrics, Rio de Janeiro, v. 81, n.4, p.332-336. 2005. doi: 10.2223/JPED.1372

PRINCE, R.;DEVINE, A.;DICK, I.;CRIDDLE, A.;KERR, D.;KENT, N.;PRICE, R. ;RANDALL, A. The effects of calcium supplementation (milk powder or tablets) and exercise on bone density in postmenopausal women. Journal of Bone and Mineral Research, New York, v. 10, p.10681075. 1995. doi: 10.1002/jbmr.5650100711

SHIGUEMOTO, G. E.;ROSSI, E. A.;BALDISSERA, V.;GOUVEIA, C. H.;DE VALDEZ VARGAS, G. M. ;DE ANDRADE PEREZ, S. E. Isoflavone-supplemented soy yoghurt associated with resistive physical exercise increase bone mineral density of ovariectomized rats. Maturitas, Amsterdam, v. 57, n.3, Jul 20, p.261-70. 2007. doi: 10.1016/j.maturitas.2007.01.011

SIQUEIRA, F. V.;FACCHINI, L. A.;AZEVEDO, M. R.;REICHERT, F. F.;BASTOS, J. P.;SILVA, M. C.;DOMINGUES, M. R.;DUMITH, S. C. ;HALLAL, P. C. Prática de atividade física na adolescência e prevalência de osteoporose na idade adulta.

Revista Brasileira de Medicina do Esporte, Niterói, v. 15, n.1, p.27-30. 2009. doi: 10.1590/S1517-86922009000100006

TENÓRIO, A. D. S.;ALVES, S. B.;BEZERRA, A. D. L.;SOUZA, G. M. L.;CASTANHO, M. T. J. A.;TASHIRO, T.;GALINDO, L. C. M. ;MORAES, S. R. A. Efeito do treinamento físico sobre o tecido ósseo e a concentracão sérica de cálcio em camundongos fêmeas ovariectomizadas.

Acta Cirurgica Brasileira, São Paulo, v. 20, n.4, p.280-283. 2005. doi: 10.1590/S010286502005000400004

WELCH, J. M. ;WEAVER, C. M. Calcium and exercise affect the growing skeleton. Nutr Rev, Baltimore, v. 63, p.361-373. 2005. url: http://www3.interscience.wiley.com/journal/11982 1030/abstract?CRETRY $=1 \&$ SRETRY $=0$. Acesso em 17/02/2010

ZEHNACKER, C. H. ;BEMIS-DOUGHERTY, A. Effect of weighted exercises on bone mineral density in post menopausal women. A systematic review. Journal of Geriatric Physical Therapy, La Crosse, v. 30, n.2, p.79-88. 2007.

Endereço:

Raphael Mendes Ritti Dias

Upe - Escola Superior de Educação Física

Rua Arnóbio Marques, 310 Santo Amaro

Recife PE Brasil

50100-130

e-mail: raphael.dias@upe.br

Recebido em: 03 de agosto de 2009.

Aceito em: 23 de fevereiro de 2010.

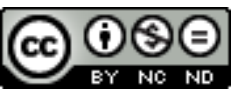

Motriz. Revista de Educação Física. UNESP, Rio Claro, SP, Brasil - elSSN: 1980-6574 - está licenciada sob Licenca Creative Commons 